\title{
Hereditary Podocytopathies in Adults: The Next Generation
}

\author{
Olivia Boyer $^{\mathrm{a}, \mathrm{c}}$ Guillaume Dorval ${ }^{\mathrm{a}, \mathrm{c}}$ Aude Servais ${ }^{\mathrm{b}, \mathrm{c}}$ \\ ${ }^{a}$ Néphrologie pédiatrique and ${ }^{\mathrm{b}}$ Néphrologie, Centre de référence MARHEA, Hôpital Necker - Enfants Malades, \\ APHP, and ${ }^{b}$ Inserm U1163, Institut Imagine, Université Paris-Descartes Sorbonne Paris Cité, Paris, France
}

\section{Keywords}

Familial nephrotic syndrome $\cdot$ Genetics S Steroid resistance $\cdot$ Podocyte $\cdot$ Next-generation sequencing

\begin{abstract}
Idiopathic nephrotic syndrome may have two underlying mechanisms: either (1) an alteration of the immune system resulting in the production of a putative circulating factor of glomerular permeability; or (2) mutations in the structural genes of the glomerular filtration barrier in which case patients are typically multidrug resistant and do not recur after transplantation. The latter forms have been recently recognized as "hereditary podocytopathies." In the past few years, positional cloning approaches that allow the identification of gene mutations underlying diseases whose pathophysiology is unknown and animal models have helped decipher the pathophysiological mechanisms of the glomerular filtration process. Recently, the advent of next-generation sequencing (NGS) techniques has greatly facilitated the identification of numerous novel causative genes in hereditary podocytopathies. Moreover, it has revealed mutations in unexpected genes and has widened the phenotypes associated with podocyte gene mutations. The list of genes mutated in hereditary podocytopathies is constantly evolving and consists to date of more than 40 genes. However, the most recently identified genes are extremely rarely mutated and
\end{abstract}

\section{KARGER}

(C) 2017 S. Karger AG, Basel

E-Mail karger@karger.com

www.karger.com/kdd may concern only a couple of families worldwide. These discoveries provided crucial insight into the pathophysiological mechanisms linking podocyte proteins to kidney function. This review will focus on monogenic podocytopathies affecting adult patients.

(c) 2017 S. Karger AG, Basel

\section{Introduction}

Idiopathic nephrotic syndrome may have two underlying mechanisms [1]: either (1) an alteration of the immune system resulting in the production of a circulating factor of glomerular permeability, yet to be identified - in which case patients are usually sensitive to immunosuppressive drugs, but may also be multidrug resistant with a high recurrence rate after renal transplantation; or (2) mutations in the structural genes of the glomerular filtration barrier. Patients are typically multidrug resistant, although partial remission may be observed with the calcineurin inhibitors ACEi and ARBs (i.e., reduced but persistent proteinuria and normal albuminemia), and do not recur after transplantation. The latter forms have been

Contribution from the International Conference: The Kidney in Genetic and Rare Diseases, Naples, October 27-29, 2016. 
recently recognized as "hereditary podocytopathies." In the past few years, positional cloning approaches that allow the identification of gene mutations underlying diseases whose pathophysiology is unknown and animal models have helped decipher the pathophysiological mechanisms of the glomerular filtration process. Recently, the advent of next-generation sequencing (NGS) techniques has tremendously facilitated the identification of numerous novel causative genes in hereditary podocytopathies. Moreover, it has revealed mutations in unexpected genes and has widened the phenotypes associated with podocyte gene mutations. The list of genes mutated in hereditary podocytopathies is constantly evolving and consists to date of more than 40 genes. However, the most recently identified genes are extremely rarely mutated and may concern only a couple of families worldwide. These discoveries provided crucial insight into the pathophysiological mechanisms linking podocyte proteins to kidney function. While the identification of mutations in the NPHS1 and NPHS2 genes, which encode nephrin and podocin, demonstrated the central role of the slit diaphragm in glomerular function $[2,3]$, the identification of ACTN4, INF2 [4], and ANLN [5] mutations, encoding a-actinin-4, INF2, and anillin, respectively [6], emphasized the importance of an intact podocyte actin cytoskeleton in kidney physiology.

This review will focus on monogenic podocytopathies affecting adult patients. Late-onset podocytopathies are less frequent than those presenting during childhood, and are characterized by a slower progression to endstage kidney disease (ESKD), incomplete penetrance, and variable expressivity, and mostly have an autosomal dominant $(\mathrm{AD})$ inheritance.

\section{Input from Positional Cloning}

Positional cloning approaches (linkage analyses, homozygosity mapping) analyze the cotransmission of a morbid trait (i.e., proteinuria or nephrotic syndrome) with microsatellite markers spread along the whole genome. Once a putative locus has been defined, candidate genes within the candidate region are sequenced. These approaches allow the direct identification of genes involved in hereditary diseases, the pathophysiology of which is unknown. They require several informative families or large consanguineous families and may be hampered by incomplete penetrance. Despite these limitations, they led to the identification of several genes whose mutations underlie hereditary podocytopathies in adults.

Hereditary FSGS
NPHS2 and the Slit Diaphragm Signaling Platform

The NPHS2 gene was identified through positional cloning by Boute et al. [3] in 2002. NPHS2 mutations are the most frequent cause of autosomal recessive steroid-resistant nephrotic syndrome starting around 4 years of age with minimal changes or FSGS lesions on biopsy, and eventually leading to ESKD by 10 years of age. Thereafter, NPHS2 mutations were identified in $42 \%$ of autosomal recessive cases but also 10-30\% of sporadic cases [7-9]. NPHS2 encodes podocin, an integral membrane protein with a hairpin-like structure located at the podocyte slit diaphragm [10]. It homodimerizes at the C-terminal domain and interacts with other key players of the slit diaphragm such as nephrin [11] or TRPC6 [12]. Its precise function remains uncertain, but podocin could play a key role in assembling slit diaphragm proteins on a signalling platform transducing extracellular signals to the podocyte cytoskeleton, thereby conferring plasticity to the podocyte.

Most mutations provoke a retention of the mutant protein in the endoplasmic reticulum $[13,14]$. This has two major clinical consequences: endoplasmic reticulum-retained variants are associated with an earlier onset of nephrotic syndrome (starting around 2 years) than mutants correctly addressed at the plasma membrane (presenting around 10 years of age) [14, 15]. Therefore, the search for pharmacological chaperones able to properly localize podocin mutants at the slit diaphragm is ongoing and seems very promising to slow the progression of the disease.

Subsequent studies revealed that NPHS2 mutations are the second cause of congenital nephrotic syndrome after nephrin gene mutations, and a leading cause of steroid-resistant nephrotic syndrome and primary FSGS in adults. Almost all adult-onset cases with NPHS2 mutations carry the p.R229Q nonneutral polymorphism associated with a pathogenic mutation on the second allele [16-18]. This variant decreases the nephrin-binding capacity of podocin [16]. Our group demonstrated that the effect of the p.R229Q polymorphism might be far more complex than expected in a Mendelian disorder [19]. Indeed, the p.R229Q variant is only pathogenic when transassociated to specific C-terminal mutations, which exert a dominant negative effect through an altered dimerization and mislocalization. On the contrary, patients bearing the p.R229Q variant at the homozygous state are asymptomatic. This has direct implications in genetic counseling since the risk of transmission of the disease may be definitely different from what is expected in autosomal recessive disorders, and also to evaluate the recurrence risk following renal transplantation $[1,19]$.

Kidney Dis 2017;3:50-56 DOI: $10.1159 / 000477243$ 
TRPC6 and the Calcium Signaling Pathway in

Podocytopathies

TRPC6 gene mutations are responsible for $5 \%$ of $\mathrm{AD}$ podocytopathies $[12,20,21]$. Affected patients present with nephrotic range proteinuria in their third or fourth decade of life and progress to ESKD within 10 years after onset. TRPC6 (Transient Receptor Potential) is a cationic channel that mediates calcium entry into cells and is involved in mechanosensation $[22,23]$. TRPC6 is expressed at the podocyte slit diaphragm, where it interacts with podocin and nephrin [12]. Several gain-of-function missense mutations cause an increase in intracellular calcium influx $[5,12]$, whereas others result in a loss-offunction phenotype [24]. This discovery unexpectedly suggested the implication of calcium signalling in the pathogenesis of FSGS.

\section{ACTN4 and the Implication of Cytoskeleton}

Components in Podocytopathies

A genome-wide scan performed in a 100-member kindred allowed Pollak's group to map the first locus of AD podocytopathies on chromosome 19q13 [25, 26]. Linkage analysis including additional families helped to reduce the size of the region and led to the identification of three missense mutations in the ACTN4 gene [27]. Subsequently, ACTN4 mutations were evaluated to be responsible for approximately $4 \%$ of AD podocytopathies [28]. Patients typically present proteinuria during their teenage years or later and reach ESKD by the age of 50 years [26-30]. ACTN4 encodes the actin-binding protein $\alpha$-actinin-4, highly expressed in podocytes. Some mutations exert a gain-of-function effect by increasing the affinity of $\alpha$ actinin- 4 to actin, and/or inducing the formation of actin aggregates around the nucleus, with subsequent impairment of podocyte motility [27, 28, 31-33]. These results underlie the crucial importance of podocyte actin cytoskeleton integrity in kidney physiology.

\section{INF2 and the Role of Formin Proteins in}

\section{Podocytopathies}

In 2010, heterozygous mutations in the INF2 gene encoding the inverted formin 2 were identified in $12 \%$ of families with AD FSGS [4], and subsequently in 17\% of pedigrees in a European cohort [34], thereby confirming that INF2 mutations are the first cause of AD podocytopathies. Age at proteinuria onset ranges from 5 to 72 years and at ESKD onset from 13 to 70 years. As most AD disorders, these mutations have an incomplete penetrance and a variable expressivity. Conversely, less than $1 \%$ of sporadic cases with FSGS are mutated [34]. Formins are large multidomain ubiquitous proteins that have essential roles in remodeling the actin and microtubule cytoskeletons [35]. INF2 belongs to the diaphanous-related formin family, members of which are direct effectors of Rho-family GTPases [36]. The majority of known INF2 mutations involve highly conserved residues of the diaphanous-inhibitory domain of the protein product, suggesting its crucial role. These data emphasize the role of a dynamic regulation of the cytoskeleton in podocyte physiology.

\section{Input from Candidate-Gene Approaches}

This approach aims to identify causative mutations in genes encoding proteins that could have a direct role in the pathophysiology of a particular disorder. It is based on scientific knowledge of the putative protein functions and interactions.

\section{INF2 Mutations in Podocytopathies with \\ Charcot-Marie-Tooth Disease}

Since the 1960s, an increased prevalence of nephropathies, particularly FSGS, has been documented in patients with Charcot-Marie-Tooth disease, a frequent inherited chronic peripheral motor and sensory neuropathy [37]. The pathophysiological mechanism linking these two clinical entities was, however, unknown. INF2 was known to interact with the Rho-GTPase Cdc42 and the myelin component MAL (Myelin and Lymphocyte protein), both implicated in essential steps of myelination and myelin maintenance. Therefore, INF2 was a good candidate to underlie this neuro-renal phenotype. Our group demonstrated that INF2 mutations are the main cause of Charcot-Marie-Tooth disease with FSGS by identifying $75 \%$ of INF2 mutations in a series of 16 affected families [34]. Conversely, no mutation of INF2 was detected in patients with Charcot-Marie-Tooth disease, and no renal involvement either. Since the original publication, several other families have been described in the literature. Affected patients typically develop proteinuria between 7 and 30 years of age with FSGS and Charcot-Marie-Tooth disease of the intermediate type (i.e., with both demyelinating and axonal lesions, and intermediate median-nerve conduction velocities) during the second decade of life. INF2 is expressed in Schwann cells, where it colocalizes and interacts with MAL, and INF2 mutations alter both the polymerization and depolymerization properties of INF2, thereby perturbing myelination. These discoveries represent a major breakthrough. Indeed, although podocytes and peripheral nervous cells are highly specialized
52

Kidney Dis 2017;3:50-56 DOI: $10.1159 / 000477243$
Boyer/Dorval/Servais 
cells with distinct functions, they share similar cellular machineries that, once disrupted, lead to renal and neurological diseases. They prompted physicians to search for proteinuria in all patients with Charcot-Marie-Tooth disease and, similarly, to consider a careful clinical neurological evaluation for patients with FSGS.

\section{ARHGAP24 and the Role of GTPases in Podocytopathies}

Using a candidate-gene approach, Akilesh et al. [38] sequenced ARHGAP24 encoding the actin-regulating protein Rho-GAP 24, highly expressed in podocytes in 310 patients, and identified a loss-of-function heterozygous mutation in one family with $\mathrm{AD}$ podocytopathy. The index case had died of ESKD at 29 years of age, and two offsprings had reached ESKD at 12 and 20 years of age, respectively. Rho-GTPases belong to a complex pathway of actin-regulating proteins that provides numerous other interesting candidate genes potentially involved in hereditary podocytopathies.

\section{Input from NGS}

Assuming that coding sequences (or exons) represent less than $2 \%$ of the genome but contain $85 \%$ of known disease-causing variants, techniques have been developed that capture all exons ("Whole Exome") and massively sequence them in parallel ("Whole Exome Sequencing") [39]. This cost-effective strategy is useful to search for new causative genes and also in routine genetic testing. However, it raises many challenges both from technical and diagnostic standpoints, and generates large variant data sets that should be filtered in silico to identify potential candidate genes, whose pathogenicity will have to be demonstrated by segregation and functional studies. It has yielded surprising results on podocytopathies, not only by identifying new genes, but also by redefining phenotypes related to known genes.

\section{ANLN and Cell-Cycle Regulating Proteins in \\ Podocytopathies}

Mutations in the ANLN gene were identified by exome sequencing combined to linkage analyses in two families with autosomal FSGS diagnosed from 9 to 69 years, with ESKD occurring between 35 and 75 years [6]. The encoded protein anillin is an actin-binding protein that plays a key role in cytokinesis, and interacts with the slit diaphragm protein $\mathrm{CD} 2 \mathrm{AP}$ and the formin $\mathrm{mDia} 2$ during cell division. Anillin is overexpressed on kidney biopsies of patients with idiopathic FSGS. In vitro, anillin mutants have an impaired binding to CD2AP and cause an abnormal podocyte motility. These results add to the expanding group of podocyte cytoskeleton genes involved in podocytopathies.

\section{ADCK4 and Mitochondrial Components in}

\section{Podocytopathies}

Using exome sequencing and homozygosity mapping, Ashraf et al. [40] identified mutations of the ADCK4 gene implicated in coenzyme $\mathrm{Q}_{10}\left(\mathrm{CoQ}_{10}\right.$, ubiquinone) biosynthesis in seven families with isolated podocytopathies. $\mathrm{CoQ}_{10}$ is a key component of the mitochondrial respiratory chain and a potent antioxidant [41]. Subsequently, $A D C K 4$ mutations were described in a large cohort with a mostly renal limited phenotype and a teenage onset, ESKD during the second decade of life, and mild neurological features or retinitis pigmentosa in only 3 of the 26 patients [42]. A dozen enzymes are required for $\mathrm{CoQ}_{10}$ synthesis, the mutation of which leads to heterogeneous multisystemic disorders associating myopathy, seizures, ataxia, deafness, optic atrophy, and cardiomyopathy, among others. COQ2, COQ6, PDSS2, or ADCK4 gene mutations are a cause of podocytopathy and altogether, mutations in these genes are identified in about $1 \%$ of SRNS cases [43]. This is a crucial diagnosis to make since early $\mathrm{CoQ}_{10}$ supplementation may improve the symptoms and, in particular, reduce proteinuria.

\section{NGS Findings and Redefinition of Known}

\section{Gene-Associated Phenotypes}

$L M X 1 B$ and Podocytopathies without Nail Patella

Syndrome

By linkage analysis and exome sequencing in a large pedigree with adult-onset AD-FSGS and no extrarenal features, a novel $L M X 1 B$ mutation was identified. This was unexpected, since $L M X 1 B$ mutations cause Nail-Patella syndrome associating dysplasia of the patellae, nails and elbows, iliac horns, glaucoma, and in some cases FSGS with specific lesions characterized by the presence of type III collagen fibrils in the glomerular basement membrane by electron microscopy [44]. Mutations in three other families were subsequently identified that involve the same domain of the encoded transcription factor $[45,46]$ and are expected to diminish the interaction between the LMX1B homeodomain and DNA molecule. These data first demonstrated that mutations in genes involved in syndromic forms of podocytopathies may also be responsible for isolated FSGS and prompted to include these genes in NGS diagnosis strategies.

Kidney Dis 2017;3:50-56

Hereditary FSGS 
Collagen IV Genes in Podocytopathies

Similarly, COL $4 A 3$ variants were first identified by exome sequencing in children with steroid-resistant nephrotic syndrome, and subsequently COL $4 A 3$ and COL4A4 mutations were found in $10-12 \%$ of patients with AD FSGS, mostly diagnosed during adulthood [47]. Subsequent reports confirmed that COL4A3 and COL4A4 mutations are rather frequent causes of $\mathrm{AD}$ FSGS ( 10-12\%) [48, 49]. Mutations in these genes encode the $\alpha 3$ and 4 chains of collagen IV, respectively, and are responsible for Alport syndrome. Dominant forms of the disease have a high degree of phenotypic variability with inconstant gross hematuria and hearing loss [50]. None of the patients had characteristic lesions suggestive of Alport syndrome by electron microscopy. Nevertheless, all patients with available data had associated hematuria. These data emphasize the need to perform COL $4 A 3$ and COL4A4 gene screening in familial FSGS, especially in adult patients with or without microscopic hematuria or deafness.

Kidney Development Genes in Podocytopathies

WT1 encodes a transcription factor that plays an essential role in kidney and genital tract development [51, 52]. WT1 mutations cause a wide spectrum of syndromes of AD inheritance [53]: Denys-Drash syndrome in case of exon 8 and 9 mutations (male pseudohermaphroditism, diffuse mesangial sclerosis, and predisposition to Wilms' tumors) and Frasier syndromes related to specific splice site mutations in exon 9 (FSGS and male pseudohermaphroditism with increased susceptibility to gonadoblastomas in 46,XY patients) [54, 55]. Surprisingly, WT1 mutations were identified in pedigrees with isolated $\mathrm{AD}$ FSGS, including males without genital abnormalities or developmental tumors who transmitted the mutation, as observed through classical Sanger sequencing and more recently exome sequencing [11, 56-59]. These results suggest to perform WT1 testing in females with sporadic FSGS but also in males with familial AD FSGS.

PAX2 mutations were also identified by exome sequencing in seven families with AD FSGS, mostly diagnosed in the second to fourth decades of life [60]. Heterozygous mutations of $P A X 2$ were thus far known to cause congenital anomalies of the kidney and urinary tract (CAKUT), as well as papillorenal syndrome (renal hypodysplasia and optic nerve coloboma). Mutated patients may have either normal kidney ultrasound or echoic or small kidneys, dilated renal pelvis or calices [60]. Most patients do not have any extrarenal symptom. PAX2 is a transcription factor expressed during the development of the kidney as well as the otic and optic vesicles and the hindbrain. PAX2 mutations could result in FSGS secondary to nephron loss, but through dysregulation of target genes such as the transcription factor WT1 [60].

\section{Ciliary Genes in Podocytopathies}

Mutations of TTC21B encoding the ciliary retrograde intraflagellar transport-A protein IFT139 were initially identified as a cause of nephronophthisis [61]. Unexpectedly, its most common mutation (p.P209L) was identified by exome sequencing combined to linkage analyses in families with FSGS, making TTC21B the first ciliary gene involved in a glomerular disorder. The related phenotype associates late-onset proteinuria in teenage years, high blood pressure, ESKD in adulthood, and both FSGS lesion and tubular basement membrane thickening on kidney biopsies [61].

These findings modify our understanding of hereditary kidney diseases, previously classified as either "primary glomerular" or "primary tubulo-interstitial" disorders, and opens a new chapter in nephrology textbooks on "primary tubulo-glomerular" diseases.

\section{Conclusions}

NGS techniques have greatly facilitated the screening of podocyte gene mutations $[43,62,63]$ and have revealed that hereditary forms of podocytopathies are far more common than previously thought. However, causative gene mutations are identified in only a third of pediatric patients and even fewer adult patients [64]. Numerous further genes are therefore expected to be mutated in these disorders. Nevertheless, these diagnosis approaches face the difficulty of determining the causal mutation(s) amongst a great number of potential pathogenic variants. Targeted podocyte gene sequencing panels are now useful tools for fast and rather cheap genetic diagnosis, but also to overcome some ethical issues raised by exome sequencing. The constant advances in the pathophysiology of podocytopathies also open the way to therapeutic innovations. A promising therapy, still explored at a basic level, involves protein chaperones that could redirect the trafficking of missense mutant proteins to the plasma membrane when abnormally retained in the endoplasmic reticulum. Additional promising results have been obtained with drugs that stabilize the podocyte actin cytoskeleton. Podocytopathies are paradigmatic disorders to illustrate the paramount clinical relevance that genetic research can have for affected patients and families. Genet-
54

Kidney Dis 2017;3:50-56 DOI: $10.1159 / 000477243$
Boyer/Dorval/Servais 
ic diagnosis is of outmost importance for genetic counseling, to avoid ineffective and potentially harmful therapies, and even to start early suitable treatment, such as ubiquinone in $\mathrm{CoQ}_{10}$ deficiency, and hopefully in the near future, to offer specific mutation-based therapies [65-67].

\section{Conflict of Interest Statement}

The authors have no conflicts of interest to declare.

\section{References}

1 Ding WY, et al: Initial steroid sensitivity in children with steroid-resistant nephrotic syndrome predicts post-transplant recurrence. J Am Soc Nephrol 2014;25:1342-1348.

2 Kestila M, et al: Positionally cloned gene for a novel glomerular protein - nephrin - is mutated in congenital nephrotic syndrome. Mol Cell 1998;1:575-582.

3 Boute N, et al: NPHS2, encoding the glomerular protein podocin, is mutated in autosomal recessive steroid-resistant nephrotic syndrome. Nat Genet 2000;24:349-354.

4 Brown EJ, et al: Mutations in the formin gene INF2 cause focal segmental glomerulosclerosis. Nat Genet 2010;42:72-76.

5 Winn MP, et al: A mutation in the TRPC6 cation channel causes familial focal segmental glomerulosclerosis. Science 2005;308:18011804.

6 Gbadegesin RA, et al: Mutations in the gene that encodes the F-actin binding protein anillin cause FSGS. J Am Soc Nephrol 2014;25: 1991-2002.

7 Caridi G, et al: Broadening the spectrum of diseases related to podocin mutations. J Am Soc Nephrol 2003;14:1278-1286.

8 Ruf RG, et al: Patients with mutations in NPHS2 (podocin) do not respond to standard steroid treatment of nephrotic syndrome. J Am Soc Nephrol 2004;15:722-732.

9 Weber S, et al: NPHS2 mutation analysis shows genetic heterogeneity of steroid-resistant nephrotic syndrome and low post-transplant recurrence. Kidney Int 2004;66:571579.

10 Roselli S, et al: Podocin localizes in the kidney to the slit diaphragm area. Am J Pathol 2002; 160:131-139.

11 Zhu C, et al: A familial WT1 mutation associated with incomplete Denys-Drash syndrome. Eur J Pediatr 2013;172:1357-1362.

12 Reiser J, et al: TRPC6 is a glomerular slit diaphragm-associated channel required for normal renal function. Nat Genet 2005;37:739744.

13 Nishibori Y, et al: Disease-causing missense mutations in NPHS2 gene alter normal nephrin trafficking to the plasma membrane. Kidney Int 2004;66:1755-1765.

14 Roselli S, et al: Plasma membrane targeting of podocin through the classical exocytic pathway: effect of NPHS2 mutations. Traffic 2004; 5:37-44.
15 Hinkes B, et al: Specific podocin mutations correlate with age of onset in steroid-resistant nephrotic syndrome. J Am Soc Nephrol 2008; 19:365-371.

16 Tsukaguchi $\mathrm{H}$, et al: NPHS2 mutations in late-onset focal segmental glomerulosclerosis: R229Q is a common disease-associated allele. J Clin Invest 2002;110:1659-1666.

17 Machuca E, et al: Clinical and epidemiological assessment of steroid-resistant nephrotic syndrome associated with the NPHS2 R229Q variant. Kidney Int 2009;75:727-735.

18 Tonna S, et al: The R229Q mutation in NPHS2 may predispose to proteinuria in thin-basement-membrane nephropathy. Pediatr Nephrol 2008;23:2201-2207.

19 Tory K, et al: Mutation-dependent recessive inheritance of NPHS2-associated steroid-resistant nephrotic syndrome. Nat Genet 2014; 46:299-304.

20 Heeringa SF, et al: A novel TRPC6 mutation that causes childhood FSGS. PLoS One 2009; 4:e7771.

21 Santin S, et al: TRPC6 mutational analysis in a large cohort of patients with focal segmental glomerulosclerosis. Nephrol Dial Transplant 2009;24:3089-3096.

22 Clapham DE: TRP channels as cellular sensors. Nature 2003;426:517-524.

$23 \mathrm{Kriz}$ W: TRPC6 - a new podocyte gene involved in focal segmental glomerulosclerosis. Trends Mol Med 2005;11:527-530.

24 Riehle M, et al: TRPC6 G757D loss-of-function mutation associates with FSGS. J Am Soc Nephrol 2016;27:2771-2783.

25 Mathis BJ, Calabrese KE, Slick GL: Familial glomerular disease with asymptomatic proteinuria and nephrotic syndrome: a new clinical entity. J Am Osteopath Assoc 1992;92: 875-880, 883-884.

26 Mathis BJ, et al: A locus for inherited focal segmental glomerulosclerosis maps to chromosome 19q13. Kidney Int 1998;53:282-286.

27 Kaplan JM, et al: Mutations in ACTN4, encoding alpha-actinin-4, cause familial focal segmental glomerulosclerosis. Nat Genet 2000;24:251-256

28 Weins A, et al: Mutational and biological analysis of alpha-actinin- 4 in focal segmental glomerulosclerosis. J Am Soc Nephrol 2005; 16:3694-3701.

29 Vats A, et al: Familial nephrotic syndrome: clinical spectrum and linkage to chromosome 19q13. Kidney Int 2000;57:875-881.
30 Pollak MR, Alexander MP, Henderson JM: A case of familial kidney disease. Clin J Am Soc Nephrol 2007;2:1367-1374.

31 Yao J, et al: Alpha-actinin-4-mediated FSGS: an inherited kidney disease caused by an aggregated and rapidly degraded cytoskeletal protein. PLoS Biol 2004;2:e167.

32 Michaud JL, et al: Focal and segmental glomerulosclerosis in mice with podocyte-specific expression of mutant alpha-actinin-4. J Am Soc Nephrol 2003;14:1200-1211.

33 Michaud JL, et al: FSGS-associated alpha-actinin-4 (K256E) impairs cytoskeletal dynamics in podocytes. Kidney Int 2006;70:10541061.

34 Boyer O, et al: INF2 mutations in CharcotMarie-Tooth disease with glomerulopathy. N Engl J Med 2011;365:2377-2388.

35 Chesarone MA, DuPage AG, Goode BL: Unleashing formins to remodel the actin and microtubule cytoskeletons. Nat Rev Mol Cell Biol 2010;11:62-74.

36 Goode BL, Eck MJ: Mechanism and function of formins in the control of actin assembly. Annu Rev Biochem 2007;76:593-627.

37 Lemieux G, Neemeh JA: Charcot-MarieTooth disease and nephritis. Can Med Assoc J 1967;97:1193-1198.

38 Akilesh S, et al: Arhgap24 inactivates Rac1 in mouse podocytes, and a mutant form is associated with familial focal segmental glomerulosclerosis. J Clin Invest 2011;121:4127-4137.

$39 \mathrm{Ng} \mathrm{SB}$, et al: Targeted capture and massively parallel sequencing of 12 human exomes. Nature 2009;461:272-276.

40 Ashraf S, et al: ADCK4 mutations promote steroid-resistant nephrotic syndrome through CoQ10 biosynthesis disruption. J Clin Invest 2013;123:5179-5189.

41 Emma F, et al: Renal involvement in mitochondrial cytopathies. Pediatr Nephrol 2012; 27:539-550.

42 Korkmaz E, et al: ADCK4-associated glomerulopathy causes adolescence-onset FSGS. J Am Soc Nephrol 2016;27:63-68.

43 Sadowski CE, et al: A single-gene cause in $29.5 \%$ of cases of steroid-resistant nephrotic syndrome. J Am Soc Nephrol 2015;26:12791289.

44 Dreyer SD, et al: Mutations in LMX1B cause abnormal skeletal patterning and renal dysplasia in nail patella syndrome. Nat Genet 1998; 19:47-50. 
45 Edwards N, et al: A novel LMX1B mutation in a family with end-stage renal disease of "unknown cause." Clin Kidney J 2015;8:113-119.

46 Boyer $\mathrm{O}$, et al: LMX1B mutations cause hereditary FSGS without extrarenal involvement. J Am Soc Nephrol 2013;24:1216-1222.

47 Malone AF, et al: Rare hereditary COL4A3/ COL4A4 variants may be mistaken for familial focal segmental glomerulosclerosis. Kidney Int 2014;86:1253-1259.

48 Xie J, et al: COL4A3 mutations cause focal segmental glomerulosclerosis. J Mol Cell Biol 2014;6:498-505.

49 Lin F, et al: Whole exome sequencing reveals novel COL4A3 and COL4A4 mutations and resolves diagnosis in Chinese families with kidney disease. BMC Nephrol 2014;15:175.

50 Moriniere $\mathrm{V}$, et al: Improving mutation screening in familial hematuric nephropathies through next generation sequencing. J Am Soc Nephrol 2014;25:2740-2751.

51 Pritchard-Jones $\mathrm{K}$, et al: The candidate Wilms' tumour gene is involved in genitourinary development. Nature 1990;346:194-197.

52 Rivera MN, Haber DA: Wilms' tumour: connecting tumorigenesis and organ development in the kidney. Nat Rev Cancer 2005;5: 699-712.
53 Niaudet P, Gubler MC: WT1 and glomerular diseases. Pediatr Nephrol 2006;21:16531660.

54 Jeanpierre C, et al: Identification of constitutional WT1 mutations, in patients with isolated diffuse mesangial sclerosis, and analysis of genotype/phenotype correlations by use of a computerized mutation database. Am J Hum Genet 1998;62:824-833.

55 Klamt B, et al: Frasier syndrome is caused by defective alternative splicing of WT1 leading to an altered ratio of WT1 +/-KTS splice isoforms. Hum Mol Genet 1998;7:709-714.

56 Hall G, et al: A novel missense mutation of Wilms' Tumor 1 causes autosomal dominant FSGS. J Am Soc Nephrol 2015;26:831-843.

57 Benetti E, et al: A novel WT1 gene mutation in a three-generation family with progressive isolated focal segmental glomerulosclerosis. Clin J Am Soc Nephrol 2010;5:698-702.

58 Guaragna MS, et al: Two distinct WT1 mutations identified in patients and relatives with isolated nephrotic proteinuria. Biochem Biophys Res Commun 2013;441:371-376.

59 Coppes MJ, et al: Inherited WT1 mutation in Denys-Drash syndrome. Cancer Res 1992;52: 6125-6128.

60 Barua M, et al: Mutations in PAX2 associate with adult-onset FSGS. J Am Soc Nephrol 2014;25:1942-1953.
61 Davis EE, et al: TTC21B contributes both causal and modifying alleles across the ciliopathy spectrum. Nat Genet 2011;43:189-196.

62 Giglio S, et al: Heterogeneous genetic alterations in sporadic nephrotic syndrome associate with resistance to immunosuppression. J Am Soc Nephrol 2015;26:230-236.

63 McCarthy HJ, et al: Simultaneous sequencing of 24 genes associated with steroid-resistant nephrotic syndrome. Clin J Am Soc Nephrol 2013;8:637-648.

64 Buscher AK, et al: Mutations in podocyte genes are a rare cause of primary FSGS associated with ESRD in adult patients. Clin Nephrol 2012;78:47-53.

65 Rötig A, et al: Quinone-responsive multiple respiratory-chain dysfunction due to widespread coenzyme Q10 deficiency. Lancet 2000; 356:391-395.

66 Montini G, Malaventura C, Salviati L: Early coenzyme Q10 supplementation in primary coenzyme Q10 deficiency. N Engl J Med 2008; 358:2849-2850.

67 Hearing SF, et al: COQ6 mutations in human patients produce nephrotic syndrome with sensorineural deafness. J Clin Invest 2001;121: 2013-2024. 